\title{
Enhancement of Expression Level of Modified t-PA (TNKase) in Leishmania tarentolae by Induction System
}

\author{
Mohammad Mehdi Attarpour Yazdi ${ }^{1}$, Nikky Tofighi ${ }^{2}$, Taraneh \\ Rajaee $^{2}$, Marzieh Ghahremanlou ${ }^{2}$, Ahmad Adeli ${ }^{2}$, Azam Bolhassani ${ }^{3}$, \\ Mohammad Azizi ${ }^{2}$ and Noushin Davoudi ${ }^{2 *}$ \\ ${ }^{1}$ Department of Microbiology, Faculty of Medicine, Shahed University, Tehran, Iran; \\ ${ }^{2}$ Biotechnology Research Center, Pasteur Institute of Iran, Tehran, Iran; ${ }^{3}$ Department of \\ Hepatitis and AIDS, Pasteur Institute of Iran, Tehran, Iran
}

Received 8 April 2018; revised 5 May 2018; accepted 19 July 2018

\begin{abstract}
Background: The expression of bio-therapeutic proteins in mammalian cells, such as $\mathrm{CHO}$, attains high homogeneity related to post-translational modifications. Although $\mathrm{CHO}$ remains the most popular cell line for bestselling biotherapeutic proteins on the market, there are still drawbacks such as expensive culture media, long time line, and high drug cost. Recently, researches on a novel Leishmania protozoan system have confirmed that this low-level eukaryote could represent a competitive alternative to the mammalian cell lines. Methods: The full length of coding sequence of modified tPA TNKase (tenecteplase) was synthesized and cloned into an inducible expression vector of $L$. tarentolae T7-TR cells. Results: The expression of the construct was driven by a Tet-inducible promoter. A Leishmania secretory signal sequence was also added to the expression cassette to facilitate the release of the recombinant protein into the medium. The secretory recombinant protein was analyzed and confirmed by SDS-PAGE and Western blot analyses. The expression level of TNKase in this novel system of $L$. tarentolae was $810 \mathrm{IU} / \mathrm{mL}$ after induction, which means that the percentage of expression increases two times compared to previous models in L. tarentolae. The TNKase activity was comparable with Activase. Conclusion: Our results suggested that expressed TNK (modified tPA) is functionally compatible with Activase regarding their effect on fibrinolysis. Given the post-translational modification similarities between mammalian and L. tarentolae, it is speculated that this system is capable of producing complex proteins such as tPA similar to mammalian system, with easier manipulation and non-expensive method.
\end{abstract}

DOI: 10.29252/ibj.23.4.272

Keywords: Leishmania tarentolae, Tenecteplase, Tissue plasminogen activator

Corresponding Author: Noushin Davoudi

Biotechnology Research Center, Pasteur Institute of Iran, Pasteur Sq., 12 Farvardin St., Tehran 1316943551, Iran; Tel. \& Fax: (+98-21) 66480780;

E-mail: davoudi@pasteur.ac.ir or noushin_d@yahoo.com

Co-correspondence: Mohammad Azizi

Biotechnology Research Center, Pasteur Institute of Iran, Pasteur Sq., 12 Farvardin St., Tehran 1316943551, Iran;

E-mail: mohazizi2003@yahoo.co.uk

\section{INTRODUCTION}

$\mathrm{T}$ There are a variety of expression systems available for large-scale recombinant protein production. Despite various methodologies, there is no universally applicable expression system for producing recombinant protein. A proper expression system should be selected upon productivity level, bioactivity and chemical characteristics of produced protein and also the cost and safety of the system. As the need for quantity, purity, and quality improvement of bio-therapeutic products is growing, novel strategies for developing efficient eukaryotic cell systems become more mandatory ${ }^{[1]}$.

Technical restrictions observed in using different systems have forced biotechnologists to look for new 
feasible and easy-handling systems like E. coli ${ }^{[2]}$. However, limitations of $E$. coli expression system causes to achieve only $15 \%$ active form of mammalian proteins ${ }^{[3]}$. Saccharomyces cerevisiae is the only eukaryote considered for high-throughput applications, but the nature of post-translational modifications in yeast cells is different from those in human cells. Very limited number of these expression systems can ensure the proper folding of complex human recombinant proteins ${ }^{4]}$. Most eukaryotic expression systems based on insects, plants, and mammalians are slow for high throughput protein production, as the transcription of heterologous genes is mediated by a highly regulated RNA Polymerase II (Pol II). The feedback response between the overexpression of protein and the activity of RNA Pol II results in down-regulation of Pol II, leading to low expression yields ${ }^{[5]}$. In fact, RNA processing and RNA polymerase activity are coupled, which creates a regulatory step in the expression regulation of eukaryotic organisms to control protein overexpression $^{[6]}$. It is believed that multiple steps such as signal peptide cleavage disulfide bond formation and glycosylation ( $\mathrm{N}$ - and O-linked) are involved in post-translational modification of complex proteins (i.e., enzymes, co-enzymes, or antibodies) in bioactive form, which limits their expression ${ }^{[7]}$. These limitations were reasons to develop alternative expression systems capable of correcting post-translational modifications in recombinant proteins. Therefore, a new eukaryotic expression system with high level expression of recombinant proteins and less controlled polymerase activity and RNA processing is desirable. These objectives were achievable only in the members of the order Trypanosomatidae due to the natural uncoupling of transcription and RNA processing ${ }^{[5]}$. Among them, Leishmania tarentolae, a non-pathogenic parasite, has recently been investigated and employed as a potential eukaryotic expression host ${ }^{[8-10]}$. To date, several successful examples of using $L$. tarentolae as a protein expression system have been reported by our group and others ${ }^{[9-14]}$.

Tenecteplase (TNKase, Genentech, USA) is a generic variant of tissue plasminogen activator (t-PA), created by recombinant DNA technology from a mammalian cell line. Like Activase, Tenecteplase is a 527-amino-acid glycoprotein with modifications at three sites of the t-PA (Activase) structure, i.e. three substitutions, at $\mathrm{T} 103$ to $\mathrm{N}$, at $\mathrm{N} 117$ to $\mathrm{Q}$, and at KHRR (296-299) to AAAA ${ }^{[15,16]}$. These substitutions have led to a longer half-life and higher fibrin specificity than t-PA and have shown slower inhibition by PA-1 in comparison with t-PA ${ }^{[17,18]}$.

TNKase or Tenecteplase is an approved drug for treatments of acute myocardial infarction and stroke $^{[19,20]}$. Herein, we chose TNKase as a protein model, which is a t-PA with longer half-life ${ }^{[17,20]}$.

This study investigated the expression of TNKase, which is a large, complex, and highly glycosylated protein in an inducible $L$. tarentolae system. We also showed that the recombinant protein has biological activity as similar as mammalian-expressed drug (Activase).

\section{MATERIALS AND METHODS}

\section{Cultivation and maintenance of $L$. tarentolae}

L. tarentolae T7-TR strain (Cat.-No. EGE-1410, Jena Bioscience, Germany), were cultivated on brain heart infusion (BHI) medium (Merck, Germany), with the addition of $15 \mu \mathrm{g} / \mathrm{L}$ of hemin (Jena Bioscience), 50 $\mathrm{IU} / \mathrm{mL}$ of penicillin, and $50 \mu \mathrm{g} / \mathrm{mL}$ of streptomycin (Jena Bioscience). As used L. tarentolae is T7-TR, the inducible host, two more antibiotics, hygromycin (50 $\mu \mathrm{g} / \mathrm{ml})$ and nourseothricin $(50 \mu \mathrm{g} / \mathrm{ml}$; Jena Bioscience), were added. Cells were cultivated in $50-\mathrm{ml}$ ventilated flasks (Orange, USA) at $26{ }^{\circ} \mathrm{C}$ in two styles: static and agitated culture. The suspension culture of $L$. tarentolae was propagated by dilution ratio of $1: 10$ to $1: 100$ into a fresh medium when it reached the stationary growth phase. After dilution, the number of cells was typically $10^{7} / \mathrm{ml}$.

\section{Construction of inducible-integrative expression vectors}

The DNA sequence of TNK was extracted from DrugBank database (www.drugbank.ca; accession no. DB00031) and was optimized upon Leishmania codon usage and synthesized commercially (Gencust Germany). The synthetic sequence was cloned into SalI/KpnI sites of an inducible LEXSY expression vector, pLEXSY-I-blecherry3 (Fig. 1). The cloning site is in front of Leishmania mexicana secretary acid phosphatase gene (lmsapl) signal peptide in vector and is in frame with the target protein, allowing the secretory expression of TNKase in Leishmania. The resulting plasmid was confirmed by PCR analysis and digestion with different enzymes.

\section{Transfection of $L$. tarentolae T7-TR}

Initially, L. tarentolae T7-TR was grown as a static suspension in BHI broth as mentioned above. Afterwards, pLEXSY-I-blecherry3-TNKase plasmid was digested with $S w a I$ restriction enzyme (Jena Bioscience), and the 6830 -bp fragment containing TNK gene was gel purified. For transfection, logphase parasites were resuspended in $400 \mu \mathrm{l}$ of ice-cold electroporation buffer (20 mM of HEPES, $137 \mathrm{mM}$ of 


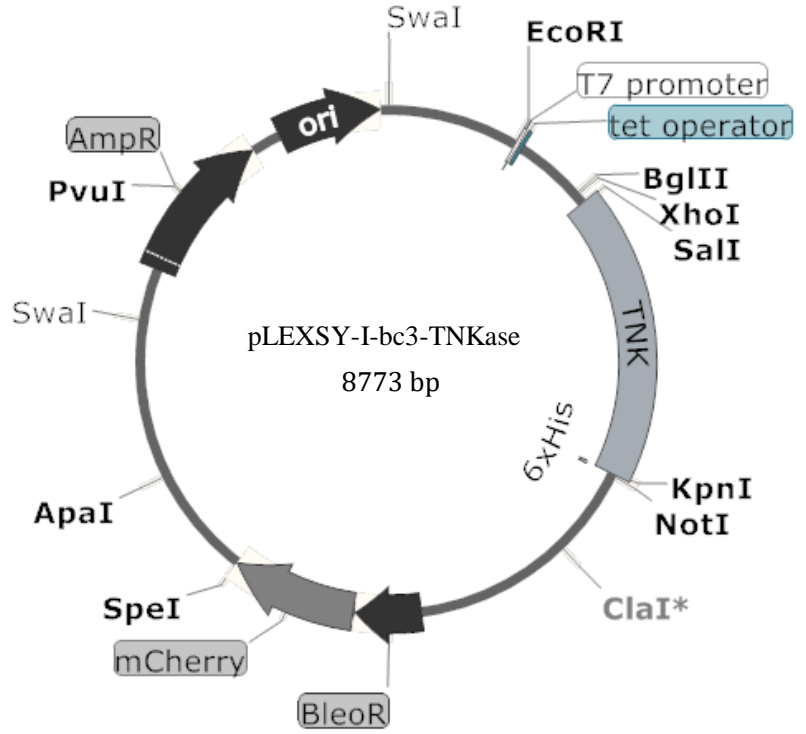

Fig. 1. Map of the pLEXSY-I-blecherry3 vector, containing TNKase gene.

$\mathrm{NaCl}, 5 \mathrm{mM}$ of $\mathrm{KCl}, 0.7 \mathrm{mM}$ of $\mathrm{Na}_{2} \mathrm{HPO}_{4}$, and $6 \mathrm{mM}$ glucose, $\mathrm{pH}$ 7.5), mixed with approximately $10 \mu \mathrm{g}$ of the linearized developed expression cassette and then electroporated using Bio-Rad Gene Pulser (Bio-Rad, USA), at $500 \mu \mathrm{F}$ and $450 \mathrm{~V}$. The electroporated promastigotes were incubated in BHI broth medium at $26{ }^{\circ} \mathrm{C}$ for $24 \mathrm{~h}$ without any drug. The selection of single colonies was done by growth on solidified BHI medium containing $50 \mu \mathrm{g} / \mathrm{ml}$ of nourseothricin, 50 $\mu \mathrm{g} / \mathrm{ml}$ of hygromycin, and $60 \mu \mathrm{g} / \mathrm{ml}$ of bleomycin (Jena Bioscience). After one week, transfected clones were appeared on solid media. Each clone was cultured and verified by PCR using TNKase specific primers (Table 1). To confirm the homologus recombination integration of the cassette containing TNKase into the odc locus of $L$. tarentolae genome in transfected strains, different diagnostic PCRs were performed. For this purpose, primer pairs, including one primer hybridizing within the expression cassette and one primer hybridizing to an odc sequence outside the developed plasmid, were applied. The information of primers is shown in Table 1. Genomic DNA from a 10$\mathrm{ml}$ culture (OD $\sim 2$ ) was extracted by conventional phenol/chloroform extraction. The culture supernatant or precipitated $L$. tarentolae cells samples were subjected to SDS-PAGE analysis. The samples were mixed with SDS sample buffer $(100 \mathrm{mM}$ of Tris- $\mathrm{HCl}$, $\mathrm{pH} 6.8,20 \%[\mathrm{v} / \mathrm{v}]$ glycerol, $2 \%[\mathrm{w} / \mathrm{v}] \mathrm{SDS}, 0.1 \%[\mathrm{w} / \mathrm{v}]$ Bromophenol blue, and $200 \mathrm{mM}[\mathrm{v} / \mathrm{v}]$ of $\beta$ mercaptoethanol) and boiled for 3 minutes. Samples from both wild and transgenic $L$. tarentolae were separated on $12 \%$ (w/v) SDS-PAGE gel. For Western blot, the resolved proteins were transferred to the nitrocellulose blotting membrane (GE Healthcare, Germany) using a wet blotting system. The membrane was blocked two $\mathrm{h}$ at room temperature with $5 \%(\mathrm{w} / \mathrm{v})$ skim milk in PBS containing $0.05 \%$ Tween-20 and then incubated for two $\mathrm{h}$ with $1 / 1,000$ dilution of antitPA antibody (rabbit polyclonal antibody to tPA, Abcam, UK). After washing, the membrane was incubated in a 1/3,000 dilution of goat anti-rabbit $\operatorname{IgG}$ horseradish peroxidase (ab6721 Abcam) at room temperature for $2 \mathrm{~h}$. Then target protein bands were developed by ECL kit (GE Healthcare, Norway).

\section{Bioactivity assay}

The plasminogen activation activity of expressed TNKase was assessed using agarose-fibrin plates according to the methods described previously with modifications ${ }^{[19,21]}$. The agarose fibrin plates were prepared as follows: $1.0 \mathrm{IU}$ of thrombin, $0.75 \mathrm{~g}$ of plasminogen, and $30 \mathrm{IU}$ of human fibrinogen were added to $12 \mathrm{ml}$ of $1.0 \%$ agarose gel that had been dissolved in normal saline at $45-55{ }^{\circ} \mathrm{C}$. The mixture was incubated at room temperature for $30 \mathrm{~min}$. The sample was then loaded in wells made onto the plate, then incubated at $37{ }^{\circ} \mathrm{C}$ for $2 \mathrm{~h}$. To determine the activity of the rTNKase, the standard tPA (Activase) was diluted and spotted onto the fibrin plate. Antibody against TPA was used for detecting the inhibition of $t$ PA lysis by anti-tPA action. The activity of the rTNKase was then measured based on the diameter of the clear zone on the fibrin plates.

\section{Amidolytic activity of rTNKase}

Chromolize $^{\mathrm{TM}}$ tPA kit (Biopool, USA) is a bio-functional immunosorbent assay designed for the quantitative determination of human tPA. The assay started by adding the samples to the micro test wells containing antibodies. The wells were washed with a mild detergent, and the sample was captured by

Table 1. The primers used in this study

\begin{tabular}{llcc}
\hline Primer & Sequence & Tm & Product (bp) \\
\hline TNK Forward & 5'-ATGGATGCAATGAAGAGAGG-3' & \multirow{2}{*}{60} & \multirow{2}{*}{1600} \\
TNK Reverse & 5'-GGTCGCATGTTGTCACG-3' & & \\
3'odcFor (A708). & 5'-GGATCCACCGCATGGCCAAGTTGACCAGTG-3' & \multirow{2}{*}{60} & 2700 \\
odc Rev (P1510) & 5'-GTGCACCCATAGTAGAGGTGC-3' \\
\hline
\end{tabular}




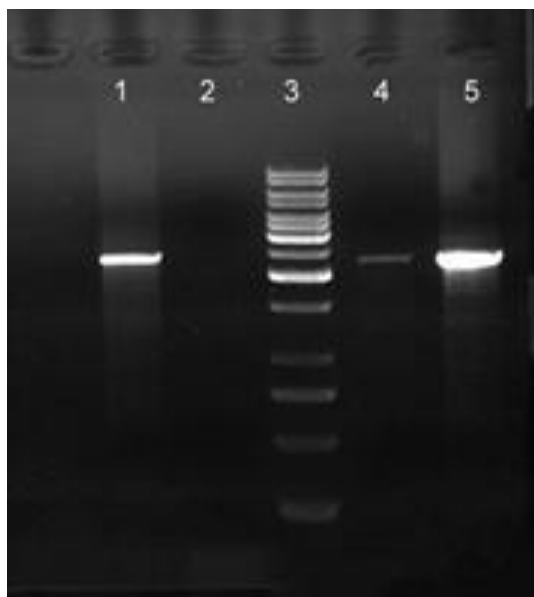

Fig. 2. Confirmation of correct integration of TNKase gene in $o d c$ site by comparison between transfected clones and nontransfected clones. All correct integration showed a 2.7-kb PCR product with two specific primers A708 and P1510. Lane1, transfected $L$. tarentolae (clone 1); lane 2, non-transfected $L$. tarentolae clone; lane 3, DNA marker (1 kb); lane 4, transfected L. tarentolae (clone 2); lane 5, transfected L. tarentolae (clone 8).

antibodies on the micro test wells. Next, the substrate consisting of plasminogen and a plasmin sensitive chromogenic substrate was added. The amidolytic activity was calculated from the increase of absorbance at $405 \mathrm{~nm}$.

\section{RESULTS}

\section{TNKase sequence}

The sequence of TNKase was optimized for codon usage of L. tarentolae (http://www.kazusa.or.jp), and then the optimized DNA sequence was synthesized . Two restriction sites, SalI and KpnI, were also added at the 5' and 3'ends, respectively. The synthetic fragment was then cloned in pLEXSY-I-blecherry3. After confirmation of the obtained construct by digestion and PCR, the recombinant pLEXSY-I-blecherry3-TNKase vector (Fig. 1) was prepared in a large scale with high purity and linearized and transfected into parasites.

\section{Transfection of construct and clonal screening and induction}

The TNKase containing fragment (6400 bp) was cut out from pLEXSY-I-blecherry3-TNKase by SwaI digestion and transformed into $L$. tarentolae by electroporation. The transfectants were screened by plating on the BHI solid medium containing bleomycin, as a selection marker. Integration of TNKase gene in the genomic DNA of the recombinant parasites was confirmed by PCR. The expected $\sim 1.6-$ $\mathrm{kb}$ band was observed in positive clones, indicating the existence of TNKase gene, and the 2.7-kb PCR product confirmed the integration of expression cassette into the odc locus of chromosome 12 of L. tarentolae (Fig. 2). For confirmatory PCR, primer pairs (Table 1), including one primer for annealing within the expression cassette as A708 and one primer hybridizing to a chromosomal odc sequence as P1510 (Jena Bioscience), were used. Two out of 20 colonies were selected for protein expression.

\section{Purification of soluble tPA protein}

To verify the expression of TNKase, the cells were cultured in BHI in a shaker incubator under induction by $10 \mathrm{mg} / \mathrm{ml}$ tetracycline. The cells were harvested at 24, 48, and $72 \mathrm{~h}$ after induction. Despite the manufacturer's suggestion, in which the best time of protein expression was $48 \mathrm{~h}$ after induction by tetracycline, TNKase expression was detectable in cell culture supernatant $24 \mathrm{~h}$ after induction and increased in time up to $72 \mathrm{~h}$. SDS-PAGE analysis on supernatant of induced culture of transfected $L$. tarentolae confirmed the high level expression of TNKase after induction related to W/O induction samples (Fig. 3A). The expression was confirmed by Western blotting in cell extracts of transgenic parasites (Fig. 3B). The dominant band of $\sim 68 \mathrm{kDa}$ was observed in culture supernatant of recombinant parasites but not in the wild-type Leishmania culture (Fig. 3B). As secreted TNKase protein contained C-terminal polyhistidine Tag, the purification was done by affinity chromatography.

\section{Characterization and activity of the recombinant TNKase}

For characterization of the recombinant TNKase, fibrin plate lysis assay was used. In order to assess whether the rTNKase was biologically active, plasminogen was used as substrate in the medium on the plate. Active TNKase was able to bind to plasminogen similar to tPA (Activase; Genentech) and to cleave it into plasmin, which degrades fibrin and results in a clear lysed zone on the fibrin/agar plate. In the center of plate, antibody against tPA (Abcam, Germany) was used. Upon the interaction of commercial antibody withthe expressed TNKase (no. 3) and standard tPA (Activase; no. 2), the immunopercipitation dark line, which is $\mathrm{Ag} / \mathrm{Ab}$ complex, was developed. Migration of samples in other wells was inhibited by $\mathrm{Ag} / \mathrm{Ab}$ complexes, i.e. transfected cell supernatant without purification (no. 4) with weak reaction related to $\mathrm{Ab}$ (no. 5) and BSA (no. 1) was used as the negative control which did not show any reaction (Fig. 4). 

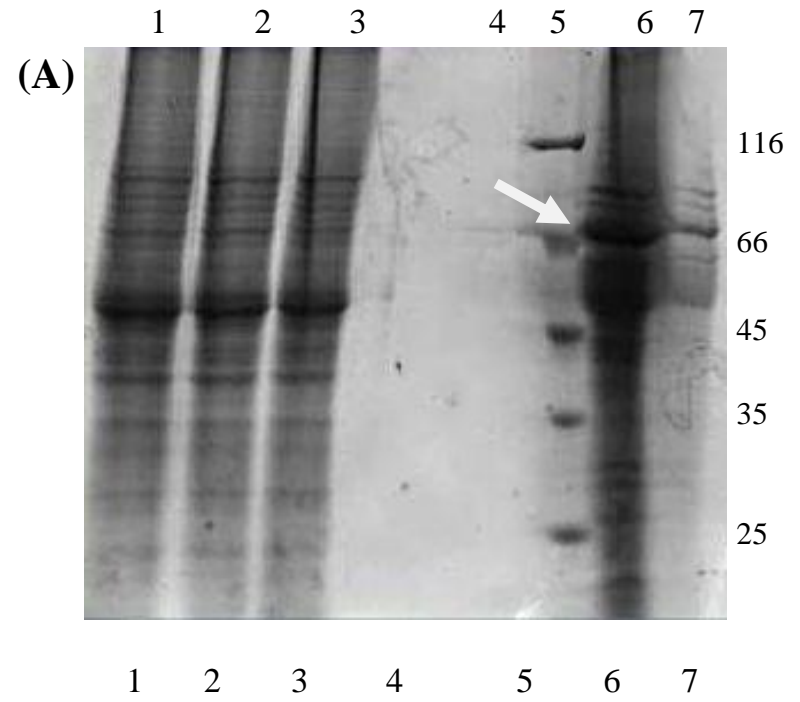

(B)

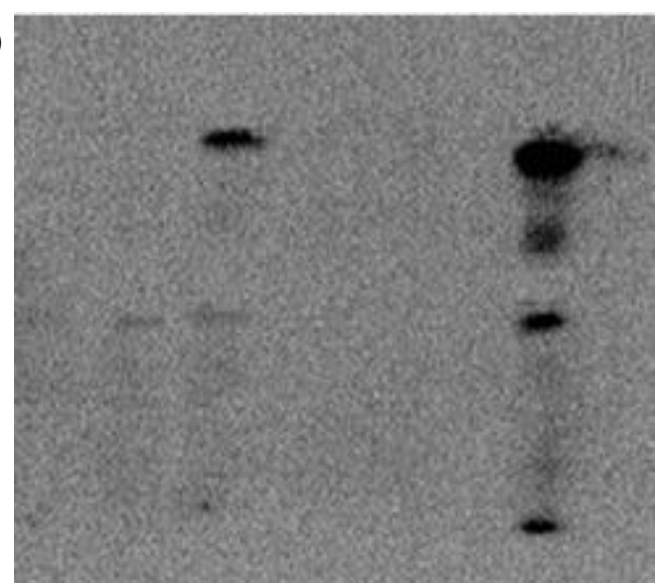

Fig. 3. SDS-PAGE (A) and Western blot (B) analyses of $L$. tarentolae culture media expressing TNK. Orders of lanes are the same in both Figures. Lane 1,30 $\mu \mathrm{g}$ of cell lysis of nontransfected L. tarentolae; lane 2, $30 \mu \mathrm{g}$ of cell lysis of transfected L. tarentolae without induction at $72 \mathrm{~h}$; lane 3, 30 $\mu \mathrm{g}$ of cell lysis of transfected L. tarentolae with induction at 72 $\mathrm{h}$; lane 4, empty well; lane 5, protein size marker (116-14 kDa); lane $6,30 \mu$ of concentrated supernatant of transfected $L$. tarentolae induced for $72 \mathrm{~h}(68 \mathrm{kDa}$ target band is indicated by arrow); lane $7,30 \mu$ l of concentrated supernatant of noninduced transfected L. tarentolae

\section{Amidolytic activity test}

Supernatant of TNKase positive cells were used for the assessment of amidolytic activity. After Affinity purification procedure, for quantitative determination of TNKase biological activity, in supernatant of transfected $L$. tarentolae, a bio-functional immunosorbent assay was performed. Expression level of transfected cells was determined during five days $(24 \mathrm{~h}$ to $110 \mathrm{~h}$ ) post culture and with different amounts of tetracycline for induction in cultures using $\mathrm{T}$ flasks with starting cell density at $1.5 \times 10^{6} \mathrm{cell} / \mathrm{ml}$. As shown in Figure 5, the highest expression level was obtained on day four. The viability of cells reduced from $95 \%$ on day one to $70 \%$ on day four. Based on Biopool Chromolize t-PA assay kit, the highest expression level was detected as $810 \mathrm{U} / \mathrm{ml}$ on day three of culture in an agitated condition and $10 \mu \mathrm{g}$ of tetracycline. Figure 5 shows the growth curve of transformed L. tarentolae in an agitated culture and the enzymatic activity of the rTNKase produced by transformed cells. The growth curves of transformed and non-transformed cells were almost identical, and therefore, only the average growth curve was plotted.

\section{DISCUSSION}

The majority of recombinant proteins in clinical use are expressed in mammalian cells, which offer native glycosylation and folding of the target proteins. Due to
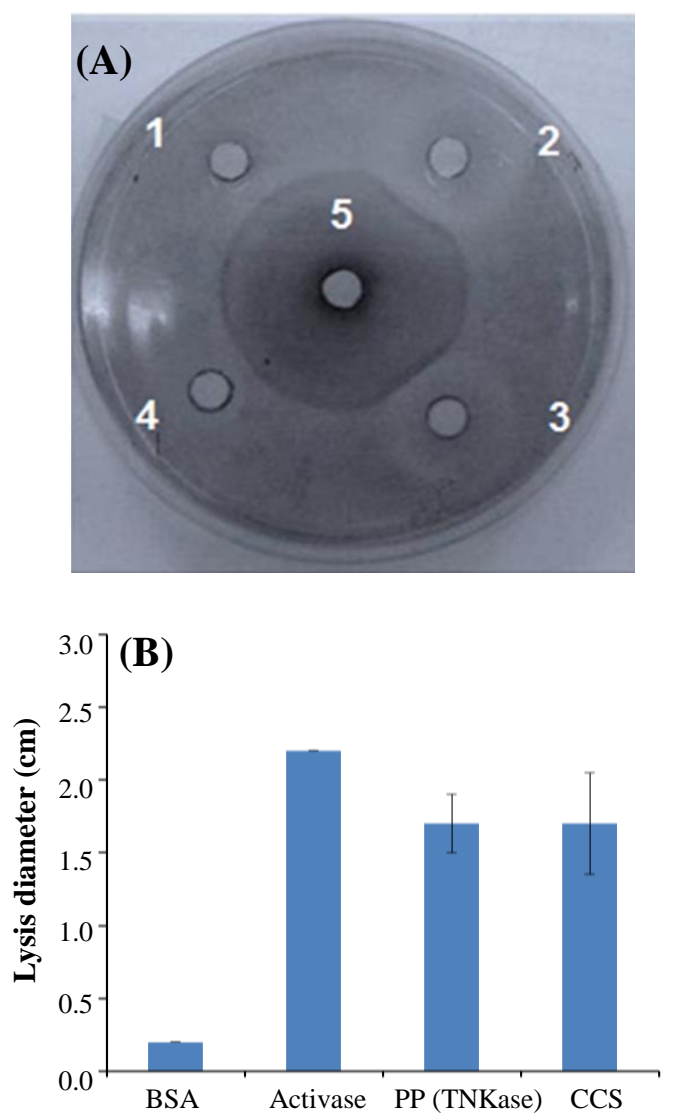

Fig. 4. Lysis on a fibrin plate using Activase and expressed rTNKase. (A) Activase standard (positive control); BSA, negative control. The fibrinolytic activity of TNKase was measured and compared with Activase (positive control) and BSA (negative control). Well 1, BSA; well 2, Activase; well 3, purified protein (TNKase); well 4, cell culture soup; well 5, Ab against t-PA (the $\mathrm{Ag} / \mathrm{Ab}$ reaction shown as dark circle around the dot). (B) the diameters of the lysis on the fibrin plate from three independent experiments. The bars indicate standard error of the mean. PP, purified protein; CCS, cell culture soup 


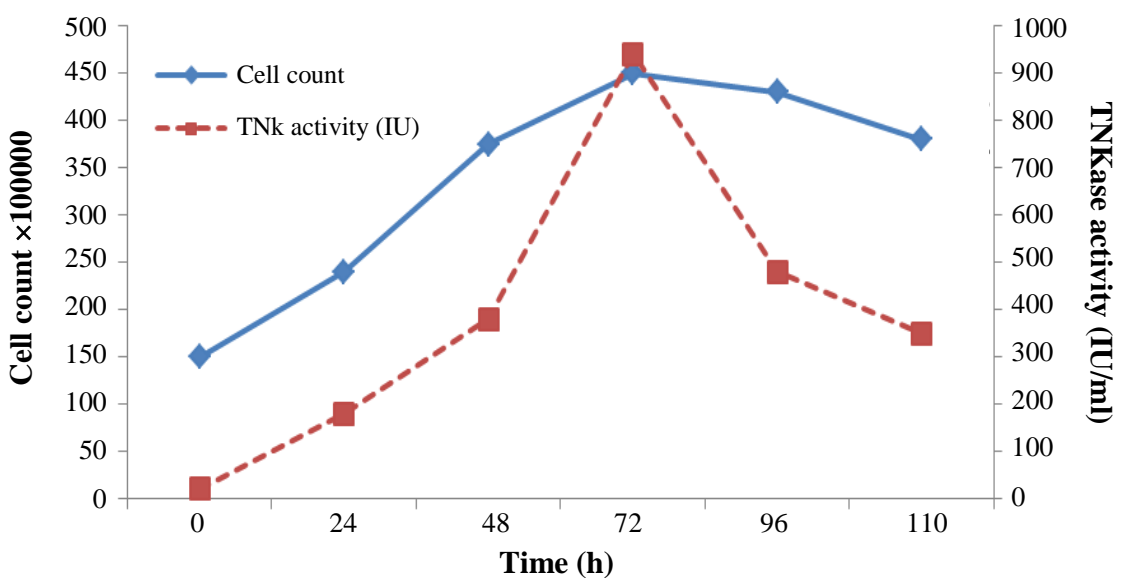

Fig. 5. Amidolytic activity of secreted TNKase transfected $L$. tarentolae culture supernatant plotted against the average cell count during a five-day agitated culture. Expression level data represent means obtained from three experiments.

incapability of prokaryotic systems in processing and secretion of complex recombinant proteins, the development of eukaryotic expression systems has proceeded in last two decades.

In eukaryotic systems such as mammalian cell, RNA Pol II is responsible for the transcription of genes in a manner of complex protein containing different transcription factors. Introduction of simple prokaryotic transcription machinery in a eukaryotic system is one of the strategies having advantage of higher system without the complex transcription regulation. Based on described strategy, a novel eukaryotic protein expression system has recently been developed in L. tarentolae ${ }^{[8]}$.

In our previous report, the expression of full length rtPA was examined in two $L$. tarentolae cell lines using different constructs without induction ${ }^{[10,12,13]}$. The synthetic cDNA of tPA, with its own signal peptide, was used for expression in $\mathrm{pFX} 1.4$ sat and pFX1.4hyg vectors. The expression level of rec-tPA in transfected L. tarentolae P10 was achieved up to 0.17 $\mu \mathrm{g} / \mathrm{ml}$ equal to $70 \mathrm{IU} / \mathrm{ml}$. Different levels of recombinant protein expression have also been reported from our and other labs, using different $L$. tarentolae expression systems ${ }^{[9-12,17,18]}$.

Expression of truncated rtPA containing only the kringle-2 and serine protease domains using
pF4splmsapx1.4 hyg vector has shown a secreted biologically active enzyme with the yield of 931 $\mathrm{IU} / \mathrm{mL}^{[13]}$. It has also been indicated that the gene dosage affects the expression level and stability of full length t-PA in L. tarentolae progeny ${ }^{[12]}$.

Based on our previous experiences, in an attempt, we examined the expression of TNKase (modified tPA) in L. tarentolae under the control of inducible T7 promoter. As it is believed that translational selection is the dominant mechanism underlying the control of differential protein expression in trypanosomatids ${ }^{[22]}$, TNKase gene sequence was designed based on trypanosome codon usage. The expression cassette also contains the acid phosphatase signal sequence of Leishmania mexicana. Using the new strategy, on day three of growth, the expression level was $810 \mathrm{IU} / \mathrm{ml}$, which was 1.7 times higher than Leishmania strain containing multicopies of t-PA gene ${ }^{[12,17]}$.

The expression level of TNKase was comparable to the truncated form. The expression was higher than full length human rtPA in L. tarentolae, $70 \mathrm{IU} / \mathrm{ml}^{[10]}$ and than multiple-copy t-PA gene integration transformant $(488 \mathrm{IU} / \mathrm{ml})^{[12]}$.

The improved level of expression can contribute to the presence of the T7/TetR system, which is less complicated comparing to mammalian expression systems (Table 2). As the secreted TNKase showed

Table 2. Different expression vectors and the level of expression of t-PA in L. tarentolae

\begin{tabular}{|c|c|c|c|c|}
\hline Protein name & $\begin{array}{l}\text { Number of construct } \\
\text { transfection }\end{array}$ & Constructs & $\begin{array}{l}\text { Expression } \\
\text { level (IU/ml) }\end{array}$ & Reference \\
\hline Complete t-PA & Doubly constructs & pFX1.4sat-tPA and pFX1.4hyg-tPA & 7 & {$[10]$} \\
\hline Complete t-PA & Doubly constructs & pFX1.4sat-2tPA and pFX1.4hyg-2tPA & $230-480$ & {$[12]$} \\
\hline Complete modified t-PA (TNKase) & Single inducible construct & pLxy-I-bleCherry3 (TNKase) & 810 & This study \\
\hline
\end{tabular}


similar activity comparing to pharmaceutical dosage form in the market, it can concluded that expressed proteins have similar folding and glycosylation pattern. The L. tarentolae expression system has several advantages in comparison to conventional protein expression systems, which include shorter doubling time (4-6 h), simple manipulation, easy handling ${ }^{[15,23]}$, and homology of the N-glycosylation pattern of $L$. tarentolae with eukaryotic glycosylation structure ${ }^{[8]}$.

In this study, transgenic inducible clones of $L$. tarentolae were developed, which was inducible by tetracycline and could express active TNKase. The functional activity of expressed TNKase was comparable with Activase. The results of our approach in expression and purification of complex proteins, which are among critical and expensive drugs, is promising and with more optimization, we expect an even higher activity and the yield of such valuable drugs.

In conclusion, the results of the present work demonstrate that the addition of $\mathrm{T} 7$ promoter/tet operator in Leishmania expression vectors can result in higher expression level of target protein. Also, codon usage optimizations of TNKase for expression in Leishmania have shown a similar result of increasing the gene dosage in high level expression. We obtained the high level of gene expression, related to the production of secretory heterologous proteins expression using inducible promoter in non-pathogen Leishmania. The novel system illustrated here could be proposed as an important alternative to mammalian expression systems which are more expensive and difficult for development.

\section{ACKNOWLEDGEMENTS}

This work has financially been supported by the National Science Foundation (INSF) Development Program (INSF ID No.92006929). We thank Dr. Esmat Mirabzadeh Ardakani, faculty member of Molecular Genetic Department at Pasteur Institute of Iran for assistance in the material preparation.

CONFLICT OF INTEREST. None declared.

\section{REFERENCES}

1. Gutierrez JM, Lewis NE, Optimizing eukaryotic cell hosts for protein production through systems biotechnology and genome-scale modeling. Biotechnology journal 2015; 10(7): 939-949.

2. Graumann K, Premstaller A. Manufacturing of recombinant therapeutic proteins in microbial systems.
Biotechnology journal 2006; 1(2): 164-186.

3. Edwards AM, Arrowsmith $\mathrm{CH}$, Christendat D, Dharamsi A, Friesen JD, Greenblatt JF, Vedadi M. Protein production: feeding the crystallographers and NMR spectroscopists. Nature structural and molecular biology 2000; 7 Suppl: 970-972.

4. Boettner M, Prinz B, Holz C, Stahl U, Lang C. Highthroughput screening for expression of heterologous proteins in the yeast Pichia pastoris. Journal of biotechnology 2002; 99(1): 51-62.

5. Kushnir S, Gase K, Breitling R, Alexandrov $\mathrm{K}$. Development of an inducible protein expression system based on the protozoan host Leishmania tarentolae. Protein expression and purification 2005; 42(1): 37-46.

6. Clancy S. RNA transcription by RNA polymerase: prokaryotes vs. eukaryotes. Nature education 2008; 1(1): 125.

7. Kumar SR. Industrial production of clotting factors: Challenges of expression, and choice of host cells. Biotechnology journal 2015; 10(7): 995-1004.

8. Breitling, R., et al., Non-pathogenic trypanosomatid protozoa as a platform for protein research and production. Protein expression and purification 2002; 25(2): 209-218.

9. Davoudi N, Hemmati A, Khodayari Z, Adeli A, Hemayatkar M. Cloning and expression of human IFN- $\gamma$ in Leishmania tarentolae. World journal of microbiology and biotechnology 2011. DOI 10.1007/ s11274-0100648-4.

10. Soleimani M, Mahboudi F, Davoudi N, Amanzadeh A, Azizi M, Adeli A, Rastegar H, Barkhodari F, MohajerMaghari B. Expression of human tissue plasminogen activator in the trypanosomatid protozoan Leishmania tarentolae. Biotechnology and applied biochemistry 2007; 48(Pt 1): 55-61.

11. Bolhassani A, Shirbaghaee Z, Agi E, Davoudi N. VLP production in Leishmania tarentolae: A novel expression system for purification and assembly of HPV16 L1. Protein expression and purification 2015; 116: 7-11.

12. Hemayatkar M, Mahboudi F, Majidzadeh-A K, Davami F, Vaziri B, Barkhordari F, Adeli A, Mahdian R, Davoudi N. Increased expression of recombinant human tissue plasminogen activator in Leishmania tarentolae. Biotechnology journal 2010; 5(11): 1198-1206.

13. Nazari R, Davoudi N. Cloning and expression of truncated form of tissue plasminogen activator in Leishmania tarentolae. Biotechnology letters 2011; 33(3): 503-508.

14. Grebowski J, Studzian M, Bartosz G, Pulaski L. Leishmania tarentolae as a host for heterologous expression of functional human ABCB6 transporter. Biochimica et biophysica acta 2016; 1858(11): 2617-2624.

15. Baruah DB, Dash RN, Chaudhari MR, Kadam SS. Plasminogen activators: a comparison. Vascular pharmacology 2006; 44(1): 1-9.

16. Nordt TK, Bode C. Thrombolysis: newer thrombolytic agents and their role in clinical medicine. Heart 2003. p. 1358.

17. Keyt BA, Paoni NF, Refino CJ, Berleau L, Nguyen H, 
Chow A, Lai J, Peña L, Pater C, Ogez J. A faster-acting and more potent form of tissue plasminogen activator. Proceedings of the national academy of sciences of the United States of America 1994; 91(9): 3670-3674.

18. Gong L, Liu M, Zeng T, Shi X, Yuan C, Andreasen PA, Huang M. Crystal structure of the Michaelis complex between tissue-type plasminogen activator and plasminogen activators inhibitor-1. The journal of biological chemistry 2015; 290(43): 25795-25804.

19. Jiao, J., M. Yu, and B. Ru, Characterization of a recombinant chimeric plasminogen activator with enhanced fibrin binding. Biochimica et biophysica acta 2001; 1546(2): 399-405.

20. Tanswell P, Modi N, Combus D, Danvas T. Pharmacokinetics and pharmacodynamics of tenecteplase in fibrinolytic therapy of acute myocardial infarction. Clinical pharmacokinetics 2002; 41(15): 1229-1245.

21. Ding FX, Yan HL, Lu YM, Xue G, Mei Q, Huang JJ, Zhao ZY, Wang YZ, Sun SH. Cloning, purification and biochemical characterization of a thrombus-ditargeting thrombolytic agent, comprised of annexin B1, ScuPA$32 \mathrm{~K}$ and fibrin-adherent peptide. Journal of biotechnology 2006; 126(3): 394-405.

22. Horn D. Codon usage suggests that translational selection has a major impact on protein expression in trypanosomatids. BMC genomics 2008; 9: 2.

23. Fritsche C, Sitz M, Weiland N, Breitling R, Pohl HD. Characterization of the growth behavior of Leishmania tarentolae: a new expression system for recombinant proteins. Journal of basic microbiology 2007; 47(5): 384-393. 